\title{
Integration of Speech Recognition-based Caption Editing System with Presentation Software
}

\author{
Kohtaroh Miyamoto, Masakazu Takizawa and Takashi Saito \\ Accessibility Center, Tokyo Research Laboratory, IBM Japan \\ Japan
}

\section{Introduction}

\subsection{Background}

Recently an increasing amount of e-Learning material including audio and presentation slides is being provided through the Internet or private networks referred to as intranets. Many hearing impaired people and senior citizens require captioning to understand such content. Captioning is a vital part of accessibility and there are national standards such as "WCAG 2.0" 1, "JIS X8341-3 5-4-d" and also laws such as "Section 508 of the Disabilities Act" 2 to assure accessibility to publicly available contents.

There are much on-going efforts for automated speech recognition enhancement, but here we will focus on the post editing to assure accurate captioning for digital archives. We introduce the method of "IBM Caption Editing System with Presentation Integration (hereafter CESPI)" which is an extension to IBM Caption Editing System (hereafter CES) ${ }^{3}$. CESPI completely includes all the functions within CES, but is further extended to include the presentation integration functions.

CES encapsulates the speech recognition engine for transcribing audio into text (CES Recorder) and also allows various editing features for error correction (CES Master and CES Client). As shown in Figure 1, CESPI integrates presentation software in various ways for both the CES Recorder and the CES Master System. Figure 2 shows a sample output of CESPI which composes of video, captioning and presentation image slide show.

\footnotetext{
1 See http://www.w3.org/TR/WCAG20/.

2 See http://www.section508.gov/.

3 See http://www.alphaworks.ibm.com/tech/ces/.
} 


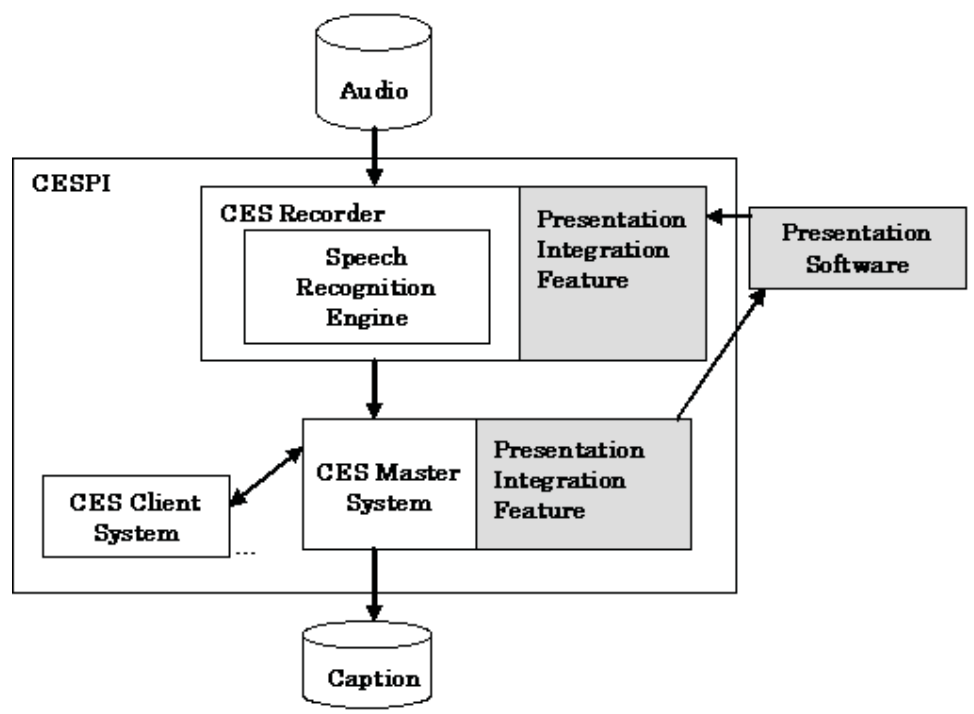

Fig. 1. CESPI receives audio input and CES Recorder by encapsulated speech recognition engine, transcribes the audio into text. CES Master System and CES Client System allows collaborative editing. CESPI adds a presentation integration feature to both CES Recorder and CES Master System.

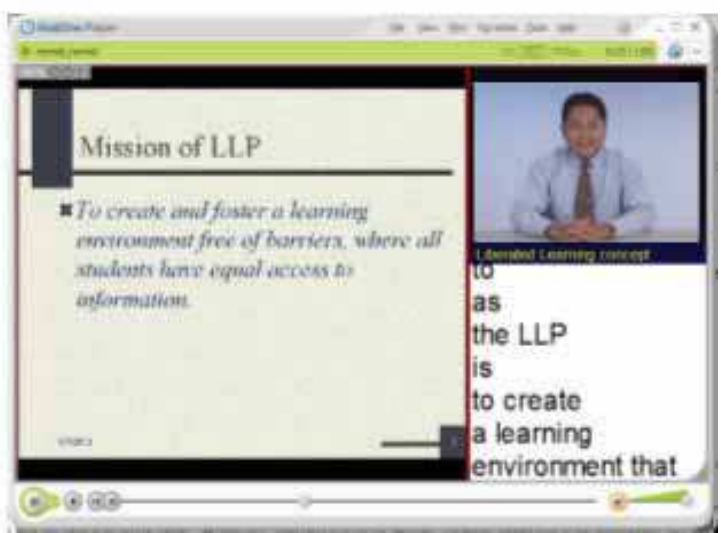

Fig. 2. The sample output of CESPI is shown. Presentation slide image is on the left hand side, video image is on the upper right hand and the caption is on the lower right hand side.

\subsection{Previous Methods}

Automatic Speech Recognition (ASR) engines have improved its accuracy ${ }^{4}$ over time. And there are many ASR related programs being introduced. Most noticeable is to use the ASR

4 McCowan et. al. (2004) discusses in detail how error rates should be handled for speech recognition results. 
for University lecture transcription. Bain et. al. (2005) introduces the ASR technology and how they are being adopted to Universities. Wald \& Bain (2008) introduces the Liberated Learning Project, Kheir \& Way (2007) introduces the VUST project, Hewitt et. al. (2008) introduces the SpeakView project. Itoh et. al. (2008) introduces the Join Project which is based on CES.

Also there are some limited efforts to use ASR for transcribing television broadcasting. Lambourne et. al. (2004) and Imai et. al. (2005) discusses the difficulties in adapting ASR to broadcasting since captioning must be completely accurate. Making corrections to the ASR transcriptions in near real-time introduces many challenges.

While most of these programs focus on the real-time transcription, there is also a strong request to transcribe the digital archives for lecturers, videos, etc. In order to create such content, it is necessary to correct the transcribed errors. Making corrections to edit these errors is a very labor intensive task (hereafter we call this task caption editing, and the system to perform the caption editing as caption editing system). Therefore our primary focus is to provide a caption editing system which is highly efficient to the user.

Goto et. al. (2007) introduces PodCastle ${ }^{5}$ which is a service available on the Internet to transcribe podcast content by ASR. And users can make corrections to erroneous words basically by selecting from ASR candidates. Also Munteanu et. al. (2008) introduces a wikilike caption editing feature to enhance the Webcast system.

\subsection{Our Prior Work}

We have introduced (Arakawa et. al. 2006) our CES technology and how it has been adopted in Universities. We previously introduced (Miyamoto 2005, Miyamoto \& Takizawa 2009) how the system can help collaborate between different roles of editors. Specifically, the master editor who is responsible for the final output uses CES Master Editing System while the client who may be any novice user uses the CES Client Editing System and both are connected by network. We also showed how the caption editing steps can be improved using three major concepts. The three concepts were "complete audio synchronization", "completely automatic audio control", and "status marking". As a result, we showed $30.7 \%$ improvement in caption editing cost.

In CES, the output phrases (as candidate caption lines) from the voice recognition engine are laid out vertically as individual lines along with timestamps. "Complete audio synchronization" means that the keyboard focus always matches the audio replay position. For example, if the audio is playing the time position of one position (e.g. 5 seconds) while the keyboard input focus is on a different position (e.g. 10 seconds), it is quite obvious that it would make it extremely difficult to make corrections to the erroneous words while listening to the audio. CES plays the audio in synchronization with the associated caption lines. This means the audio focus always matches the caption line focus.

The second concept of "completely automatic audio control", means that the audio is fully controlled automatically by the system. Users are not required to "replay" and "stop" the audio manually (usually a huge number of times). As the editing begins, the focus is set on the initial series of words, and the audio which is associated to that portion is replayed automatically. By comparing the audio with the transcribed words, user needs to determine if the words are correct. If it is, the user can press the enter key to move focus to the next

5 See http://podcastle.jp/ 
series of words, but if not the user needs to make the correction. The audio will be repeatedly replayed over again to urge the user for action. The replay stops automatically when the user types any key since it is usually annoying to hear the audio during typing. A long pause in typing will automatically restart the audio again. As a result the user does not need to operate the audio replay at all and he/she can solely concentrate on making corrections. In writing it may seem quite obvious, currently we identify that CES is the only system which has this feature.

The last concept is "status marking". The unverified lines are automatically distinguished from the corrected lines as shown in Figure 3, in CES, each caption line includes a button which is used to mark the status of each caption line. The mark also corresponds to the color of the font. The marks have several useful meanings, but basically these marks make it easier to keep track of how far the caption editing has progressed. This is very important in many cases because it is required to keep track of the caption editing work progress. And then estimate the projected finishing time and also it is needed to take appropriate action in cases such that the target deadline may be missed.

\begin{tabular}{|c|c|c|c|c|c|}
\hline \multicolumn{6}{|c|}{ File Edit View Various setting Tools Help } \\
\hline & Start Time & State & Cho & ice & \\
\hline 1 & $00: 00: 01.497$ & 0 & $\times$ & 0 & Hello. \\
\hline 2 & $00: 00: 01.896$ & 0 & $x$ & 0 & $I$ rould \\
\hline 3 & $00: 00: 02.065$ & 0 & $x$ & 0 & like to introduce \\
\hline 4 & 00:00:03.283 & $\mathrm{O}$ & $x$ & 0 & the Caption Editing \\
\hline 5 & $00: 00: 04.240$ & 0 & $x$ & 0 & Systen \\
\hline 6 & 00:00:05.039 & 0 & $x$ & 0 & or \\
\hline 7 & 00:00:05.388 & 0 & $x$ & 0 & CES for short. \\
\hline 8 & 00:00:08.900 & $\mathrm{O}$ & $x$ & 0 & Here's a brief \\
\hline 9 & $00: 00: 09.638$ & 0 & $x$ & 0 & introduction \\
\hline 10 & 00:00:10.386 & 0 & $x$ & 0 & before \\
\hline 11 & $00: 00: 10.815$ & 0 & $x$ & 0 & re go \\
\hline 12 & $00: 00: 11.155$ & 0 & $x$ & 0 & in to \\
\hline 13 & $00: 00: 11.494$ & 0 & $x$ & 0 & the details. \\
\hline 14 & $00: 00: 14.008$ & 0 & $x$ & 0 & Yoice recognition is \\
\hline 15 & $00: 00: 15.225$ & 0 & $x$ & 0 & very useful \\
\hline 16 & $00: 00: 16.044$ & 0 & $x$ & 0 & to \\
\hline 17 & $00: 00: 16.243$ & 0 & $x$ & 0 & transeribe \\
\hline 18 & $00: 00: 17.021$ & $?$ & $x$ & 0 & fordeal \\
\hline 19 & $00: 00: 17.480$ & ? & $x$ & 0 & into caption \\
\hline 20 & $00: 00: 19.645$ & $?$ & $x$ & 0 & unfortunately you \\
\hline 21 & $00: 00: 20.693$ & $?$ & $x$ & 0 & cannot expect \\
\hline 22 & $00: 00: 21.621$ & $?$ & $x$ & 0 & one hundred $X$ accuracy \\
\hline
\end{tabular}

Fig. 3. The sample image of CES is shown.

Here in this example, all of the caption lines are initially marked as "unverified" (question mark "?"). As the corrections proceed the flags are automatically converted to "determined" (circles). Here, caption lines 1 to 17 are correct since they were either correctly transcribed by the voice recognition engine or they were corrected using the editing feature in CES. Caption lines 18 and later are still unverified. 
Figure 4 illustrates how the system works from the (caption) editor's perspective. The audio is played automatically, and so the editor focuses on the audio. As soon as the editor begins to type the audio stops automatically. But when the editor is not sure and pauses the typing, the audio automatically starts to play again. The editor makes the necessary changes (and hits the enter key) then the keyboard and audio focus automatically moves to the next target line.

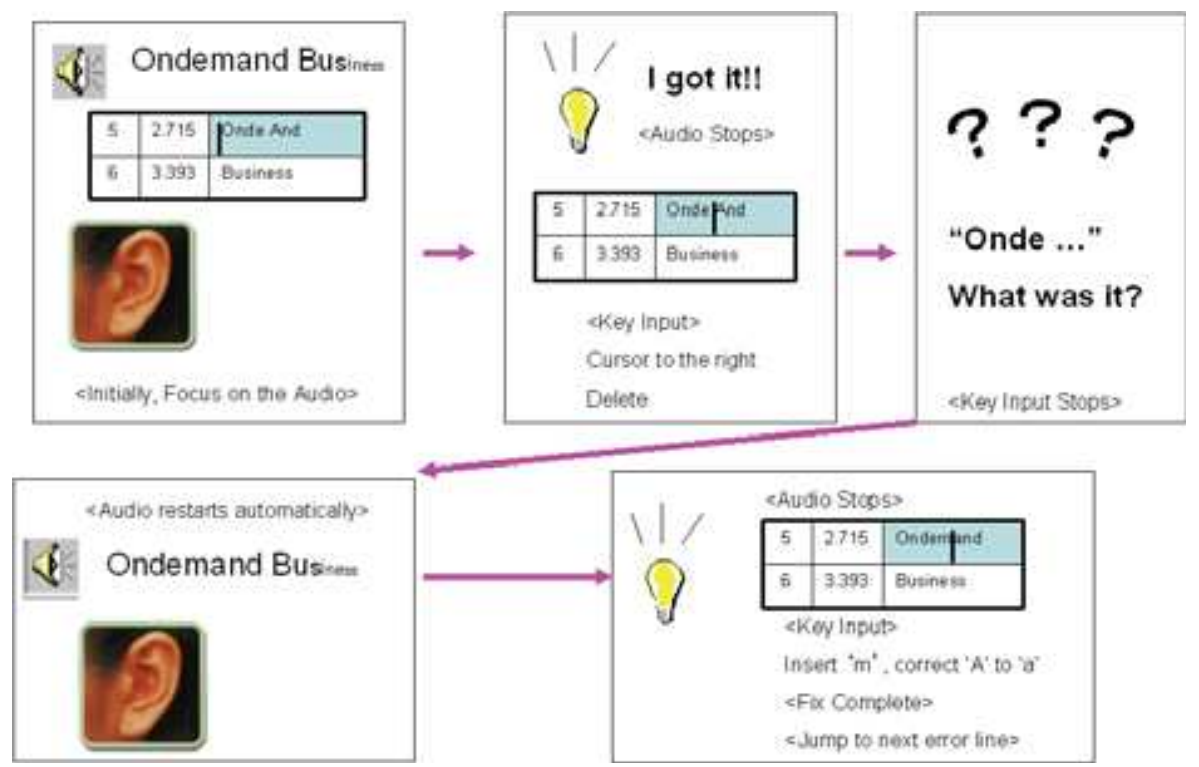

Fig. 4. The figure shows how the caption editing task using the CES. All the audio processing is automatic and user merely needs to focus on making the necessary correction.

We also showed how external scripts can help reduce the caption editing work. The experimentation results show that for example, when the recognition rate is $60.9 \%$, editing total hours decreased by 35\% (Miyamoto 2006) and the method of matching the script with the erroneous transcription results are introduced (Miyamoto 2008) as well. Furthermore, we introduced a hybrid caption editing system (Miyamoto et. al. 2007) which integrates "line unit type" (which is efficient in editing in relatively high speech recognition rate situations) with "word processor type" (which is efficient in editing in relatively low recognition rate situations). The input strings to the "word processor type" subsystem is matched to the "line unit type" subsystem. As a result of experimentation in a various speech recognition rate conditions for caption editing efficiency against previous caption editing methods, the hybrid system shows significant advantage in number of interaction and editing time. Another technique we introduced (Arakawa, et. al. 2008) the technique to make real-time automated error corrections by using confidence scores of speech recognition and automated matching algorithms of sources such as text in presentation software or scripts.

Our work goes beyond the efficiency of caption editing and also discloses the method (Miyamoto \& Ikawa 2008) to safely eliminate and dynamically distribute confidential 
information from multimedia content with audio, caption, video and presentation materials. In this chapter, we focus on the integration of Presentation software with the CES to increate the efficiency of caption editing. Presentation software provides many useful features to easily create effective e-Learning contents by the following 2 steps.

1) Prepare presentation file by combination of text, pictures, visual layout, and any other provided feature.

2) Make oral presentation using the slide show feature of the presentation software. At the same time record the movie by any video camera and/or oral presentation audio.

\section{Preliminary Survey and Investigation}

We conducted a survey to see whether the combination of video with audio, captions, and presentation slides (hereafter "multimedia composite") is helpful in understanding the content. We created 4 multimedia composites, and then allowed a total of 80 senior citizens and people with disabilities to view any content of interest freely. After viewing, we administered a survey, and asked whether the multimedia composite is useful. The results as shown in Table 1, showed that $66.3 \%$ found the multimedia composite either "Strongly Agree" or "Agree", irrelevant of age group. So we concluded that a multimedia composite is very useful for better understanding in e-Learning.

\begin{tabular}{|l|l|l|l|l|}
\hline Age Group & $\begin{array}{l}\text { Strongly } \\
\text { Agree }\end{array}$ & Agree & Disagree & $\begin{array}{l}\text { Strongly } \\
\text { Disagree }\end{array}$ \\
\hline $20 \mathrm{~s}$ & 0 & 4 & 0 & 0 \\
\hline $30 \mathrm{~s}$ & 0 & 1 & 1 & 0 \\
\hline $40 \mathrm{~s}$ & 0 & 3 & 2 & 0 \\
\hline $50 \mathrm{~s}$ & 0 & 6 & 6 & 0 \\
\hline $60 \mathrm{~s}$ & 2 & 9 & 6 & 0 \\
\hline $70 \mathrm{~s}$ & 3 & 21 & 10 & 0 \\
\hline 80 and higher & 2 & 2 & 2 & 0 \\
\hline Total & 7 & 46 & 27 & 0 \\
\hline
\end{tabular}

Table 1. Usefulness of Multimedia Composite

Next, we conducted an investigation to see whether multimedia composites are captioned. We searched through the internet for multimedia composites, and found that out of 100 composites, only 21 were adequately captioned, 1 merely provided transcript text. (Conditions were web sites free of charge, max of 5 composites per domain.) It seems that the main reason for this low rate of captioning is due to the high labor costs. There are several approaches for captioning, but here we focus on using speech recognition technology. Unfortunately the voice recognition accuracy rate is still not $100 \%$, and therefore there is still a need for an effective caption editing system to correct the errors.

The conclusion of our preliminary survey and investigation is that in order to reduce the costs of captioning content with audio and presentation slides, there is a strong need for an effective caption editing tool. The presentation slides are mostly created by commercial presentation software. In this paper, we focus on a speech recognition error correction system which integrates a caption editing system with presentation software. 


\section{Problems and Apparatus}

Based on the preliminary survey and investigation, we investigated the available caption editing tools that generate captions from audio, and identified 3 major problems. The three major problems between CES and presentation software were identified as "Content Layout Definitions", "Editing Focus Linkage", and "Exporting to Speaker Notes". To address these problems, we extended our Caption Editing System (CES) to integrate it with Microsoft PowerPoint, creating our new Caption Editing System with Presentation Integration (CESPI). The architecture in terms of code interface is shown in Figure 5.

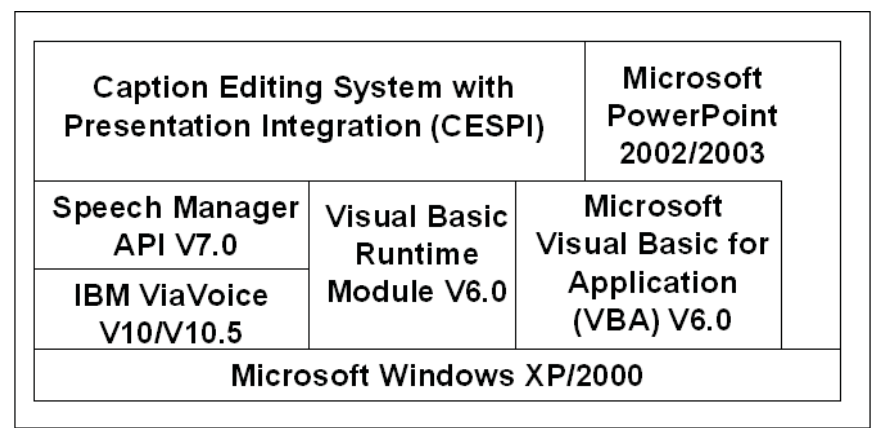

Fig. 5. The base platform is Microsoft Windows 2000/XP. User Interface of CESPI is built on Visual Basic V6.0. IBM ViaVoice engine control is implemented by Microsoft Visual C++ 6.0. The interface between ViaVoice and CESPI is Speech Manager API (SMAPI) V7.0. Also, the interface between CESPI and Microsoft PowerPoint is Visual Basic for Application (VBA) V6.0.

Finally we conducted a field test to see if the real-time transcription accuracy of state-of-theart speech recognition can show satisfactory results. We transcribed 11 University lectures in real-time and as a result obtained $81.8 \%$ accuracy. Unfortunately we have found that the speech recognition accuracy does not necessary reach the satisfaction level. There are many observations but we received many comments which required the speech recognition rate to be $85 \%$ at the least and preferably $90 \%$ for satisfactory level. So we conclude that it is required to seek a human computer transcription method to raise the accuracy (obviously without raising the cost by relying on many human resources).

\subsection{Content Layout Definition}

A multimedia composite consists of several visual components such as video, presentation images, and captions. These components needs to be laid out effectively in position and size according to such parameters as font face, font size, number of maximum characters per line, presentation image size, vice image size, resolution, overall size, and overlapping options. (Figure 6 shows a bad example of by excessive space, overlap, cut off.) CES (and CESPI) supports the RealOne Player by SMIL 6 format and also Windows Media Player by SAMI format.

\footnotetext{
6 See http://www.w3.org/AudioVideo/.
} 
The task of effectively laying out these components manually can be quite time consuming. CESPI solves this problem by automatically laying out these components based on each parameter. As shown in Figure 7, CESPI also provides a layout customization feature which allows the user to easily change the details of the layout.

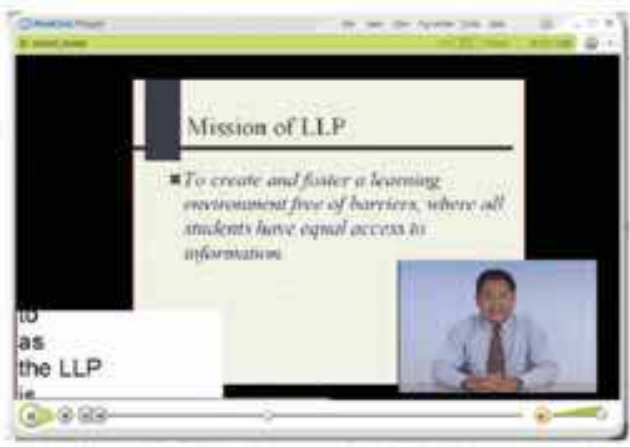

Fig. 6. The figure shows a sample of ill defined layout, where presentation image is surrounded by excessive empty space. Video and caption overlap with the presentation image. The caption is being cut off by the window boundary.

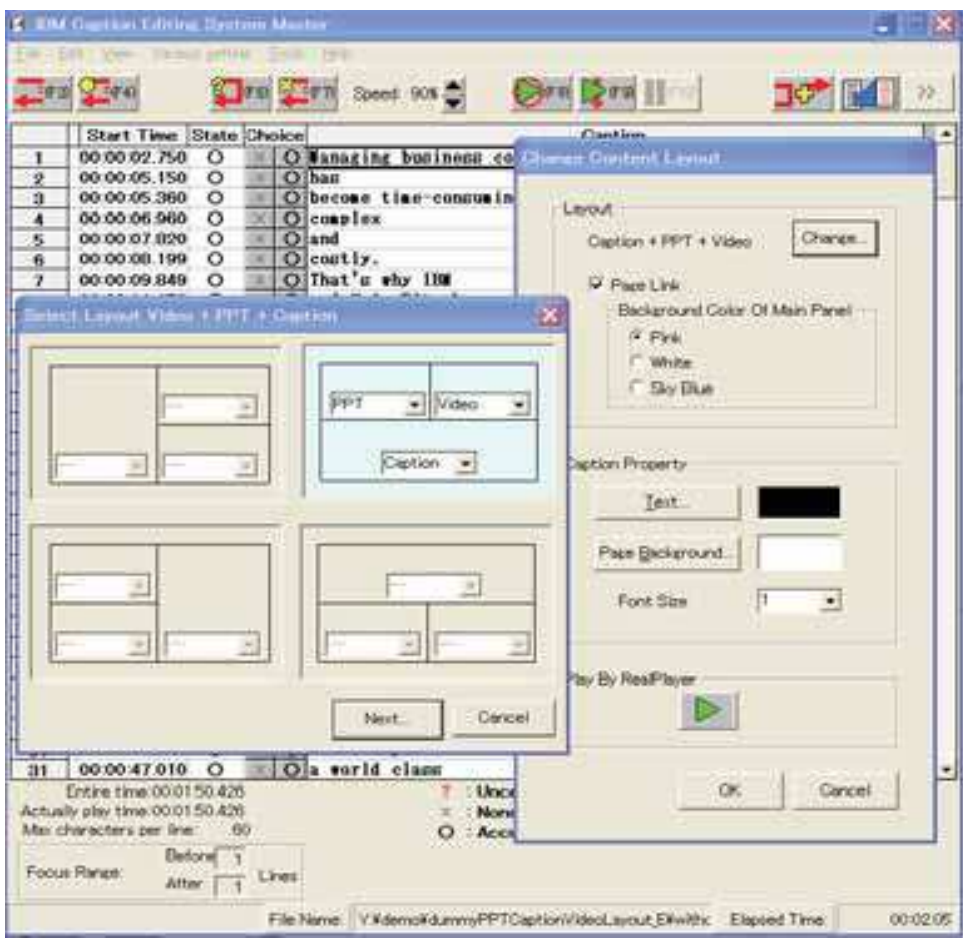

Fig. 7. The figure shows the Change Content Layout dialog on the left hand side and the Select Layout Video + PPT + Caption dialog with the focus on the right hand side 


\subsection{Editing Focus Linkage}

While editing the captions of certain multimedia composites, it is useful to reference special terminology used in the presentation slides. Because caption editing tools and presentation software were separate applications, the operating system only allows one application to have the focus at one time. Therefore it was necessary to frequently switch the focus between these two applications. Also, the user had to change to the corresponding slide pages manually. CESPI solves this problem by automatically laying out the captions, page images, and page text in a single application window, which makes it easier to view and edit the captions. CESPI also automatically interlinks between the caption timestamps and the presentation page. In other words, the presentation page always corresponds to the focused caption. (Figure 8 shows the actual user interface of the CESPI Master Editing Subsystem.)

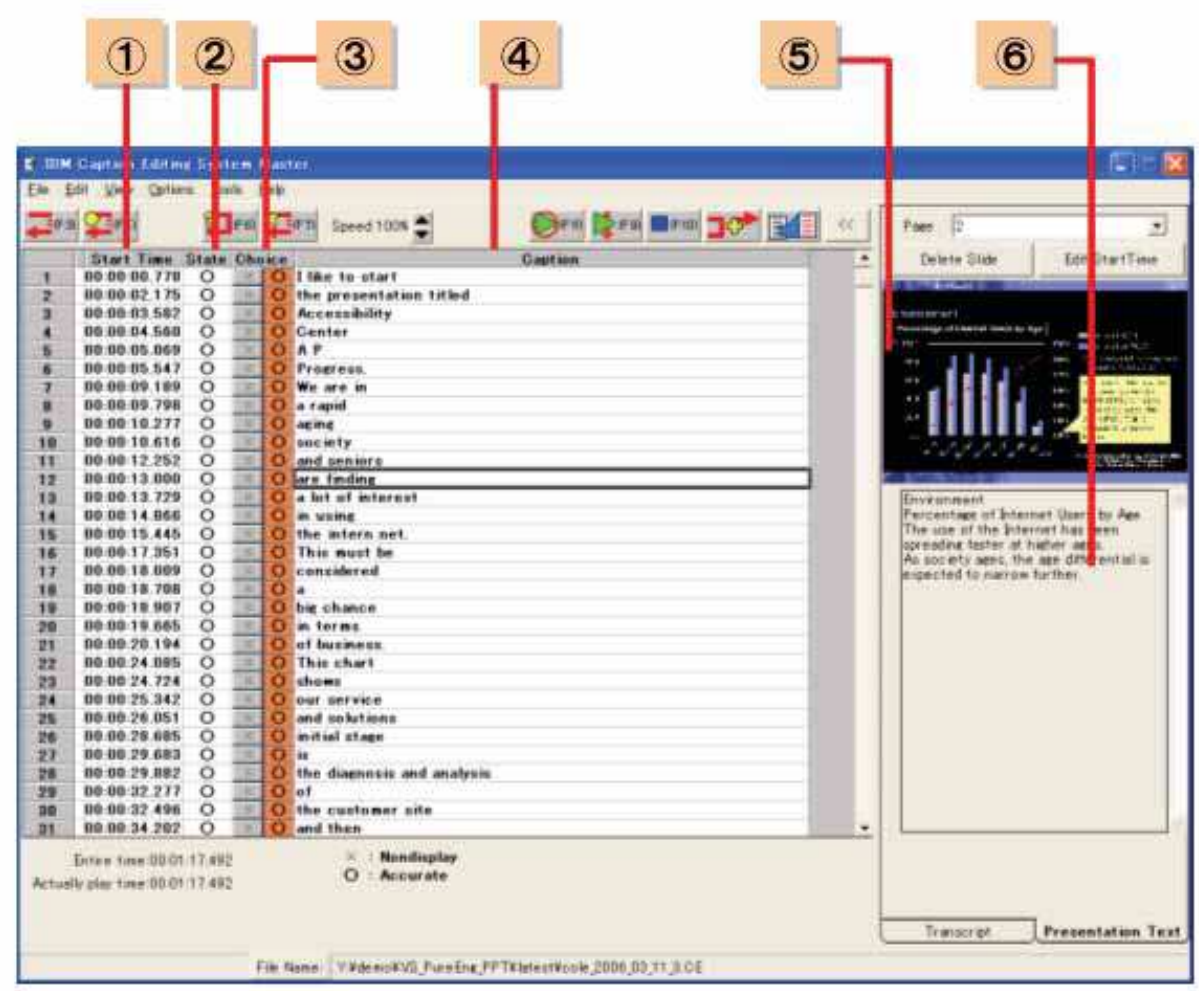

Fig. 8. On the left hand side basically shows the "Caption Line Text" (start time, state, choice, and caption). "Start Time" pointed to by the " 1 " represents the 0 based timestamp which to display the caption. "State" pointed to by the " 2 ". "Choice" is pointed to by the "3". "Caption" is pointed to by the " 4 ". On the right hand side basically shows the "Presentation Page". "Presentation Image" is pointed to by the " 5 " and retrieved text is pointed to by the " 6 ". 


\subsection{Speaker Notes Export}

Using presentation software, a speaker may define narrative notes for each presentation page (the "speaker notes"). In many cases, a single presentation package used by one presenter will be later reused by another presenter. In such cases, since the captions and speaker notes are similar, it is efficient to use the initial caption. Previously, in order to export captions to speaker notes, manual operations such as moving to the proper page and then performing copy and paste operations were required. Therefore as illustrated in Figure 9, CESPI has a capability for automatically exporting the corresponding page of the caption into the speaker notes of the presentation package.

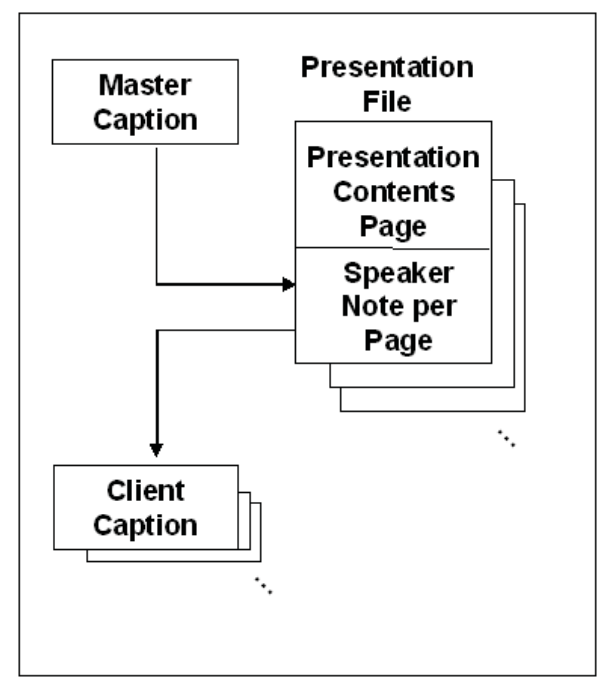

Fig. 9. Master caption is exported into the speaker notes portion of the presentation. The speaker notes can be referenced to the client caption.

\subsection{Real-time Checker and Matching}

Our technique to make automated error corrections (Arakawa, et. al. 2008) is to use confidence scores of speech recognition and also matching algorithms (to match the transcribe result with text retrieved from presentation software). Theoretically if the confidence score is high then the system can show the results of the speech recognition directly. If the confidence score is low then the system can either allow the user to make corrections or the system can try to match the result with the text retrieved from the presentation software. Unfortunately we found that the confidence score is not reliable enough to be able to detect each word's correctness by high accuracy. So here we would like to propose making use of a human resource checker. The human resource checker merely flags the transcribed result as correct or incorrect. It needs to be noted that human resource (unlike computer systems) can sometimes be very slow in processing their assigned job (which can result in a long delay). Therefore the system assures real-time captioning presence by routing the words to the matching subsystem by a higher degree as the captioning presence starts to delay. Finally, when the captioning is presented the words which were automatically corrected by the matching will be shown in blue colour and italic. 


\section{Results}

An experiment was performed to measure the editing time under the following conditions.

1. Editors are to use CES and CESPI for an approximately 30 minutes of content each.

2. It is known that as you get used to 5 editors who already have enough experience with CES and CESPI were chosen to eliminate any inconsistencies due to the learning curve effect(Barloff 1971).

3. Each editor was also assigned different portions of the content for CES and CESPI so that memory from the previous content will not take effect.

4. Task consists of correcting all the speech recognition errors, laying out the multimedia composite without each overlapping or excessive blank space, and exporting the speaker notes to the appropriate page. (Conditions are shown in Table 2.)

\begin{tabular}{|l|l|}
\hline Category & Conditions \\
\hline Window Size & $800 \times 460$ \\
\hline Component Layout Position (Video) & Right Upper Position \\
\hline Component Layout Position (Presentation Image) & Left Upper Position \\
\hline Component Layout Position (Caption) & Bottom \\
\hline Caption Font Charset & x-sjis \\
\hline Caption Font Face & osaka \\
\hline Caption Font Color & black \\
\hline Caption Font Size & +3 \\
\hline Other Conditions-1 & No Excessive Empty Space \\
\hline Other Conditions-2 & No Overlap \\
\hline Other Conditions-3 & No Cutoff \\
\hline
\end{tabular}

Table 2. Various Conditions for the Experiment

As shown in Table 3, the results showed that CESPI provided a $37.6 \%$ improvement in total editing time.

\begin{tabular}{|l|l|l|}
\hline & CESPI & CES \\
\hline Speech Recognition Rate & $81.4 \%$ & $80.8 \%$ \\
\hline Average Content Time & $28 \mathrm{~min} 24 \mathrm{sec}$ & $27 \mathrm{~min} 58 \mathrm{sec}$ \\
\hline Number of Characters & 9240 & 9221 \\
\hline Total Average Editing Time & & $127 \mathrm{~min} 2 \mathrm{sec}$ \\
\hline Editing Time Average per Content Time & $93 \mathrm{~min} 46 \mathrm{sec}$ & 4.30 \\
\hline Total Efficiency in Percentage & $37.6 \%$ & $(\mathrm{~N} / \mathrm{A})$ \\
\hline
\end{tabular}

Table 3. Result of the Experiment

Figure 10 shows the ratio of time which accounted for the saved time by "Content Layout Definition", "Editing Focus Linkage", and "Speaker Notes Export". It can be seen that

${ }^{7}$ Mainly Japanese characters, number includes punctuation marks.

${ }^{8}$ Does not include the common initial time required to listen to the whole content. 
Content Layout Definition accounted for approximately half of the time, while Editing Focus Linkage follows and then Speaker Notes Export made the slightest difference.

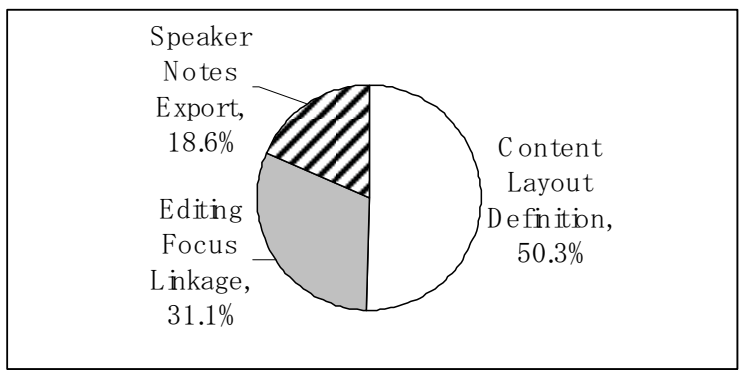

Fig. 10. Figure shows that out of the improvement of editing time shown in Table 2, 50.3\% accounted for Content Layout Definition, 31.1\% accounted for Editing Focus Linkage, 18.6\% for Speaker Notes Export.

Content Layout Definition saved much time for CESPI since content layout required much trial and error type of editing for CES. CESPI practically required almost no time since layout can be done automatically.

\section{Summary}

The three major problems between CES and presentation software were identified as "Content Layout Definitions", "Editing Focus Linkage", and "Exporting to Speaker Notes". This paper has shown how CESPI solves each of these problems. And experiment showed a $37.6 \%$ efficiency improvement compared with the previous method. Among the 3 items "Content Layout Definition" accounted for the most improvement in time, followed by "Editing Focus Linkage" and "Speaker Notes Export" came last.

Currently CESPI only supports Microsoft PowerPoint as the choice of presentation software. Future work item will be to support other presentation software.

\section{Acknowledgements}

Many people have participated in preliminary survey, so we would like to thank all those people for sparing their time.

\section{References}

Arakawa, K.; Miyamoto, K.\& Negishi, K.(2006). Caption Editing System for UniversityLevel Students with Hearing Disabilities, Closing the Gap 2006 Conference

Arakawa, K.; Miyamoto, K.\& Ohgane, T.(2008). Caption Correction Device, United States Patent Application 20080040111, International Business Machines Corporation

Bain, K.; Basson, S.; Faisman, A.\& Kanevsky, D.(2005). Accessibility, Transcription, and Access Everywhere, IBM Systems Journal: Accessibility Vol. 44 No. 3 
Baloff, N.(1971). Extensions of the Learning Curve-Some Empirical Results. Operations Research Quarterly, pp.22-44

Goto, M.; Ogata, J.\& Eto, K.(2007). PodCastle: A Web 2.0 Approach to Speech Recognition Research. in: Proceedings of the 8th Annual Conference of the International Speech Communication Association, Interspeech 2007, pp.2397-2400

Harvey, A.(2004). Nevada Legislature Develops Web Captioning System for the Hearing Impaired While technology can provide some elegant solutions, cost is still a major factor, National Association of Legislative Information Technology Newsletter

Hewitt, J.; Lyon, J.; Britton, C.\& Mellor, B.(2005). SpeakView: Live Captioning of Lectures, in: Proceedings of Universal Access in Human Computer Interaction Vol. 8, HCI International 2005

Imai, T.; Sato, S.; Homma, S.; Onoe, K.\& Kobayashi, A.(2007). Online Speech Detection and Dual-Gender Speech Recognition for Captioning Broadcast News, IEICE Trans. Inf. \& Syst., Vol. E90-D, No. 8, pp.1286-1291

Itoh, E.; Asahi, Y.\& Aoki, T.(2008). Lecture Assisting Used by Speech Recognition for the University Students with Disabilities - JOIN Project, Trace of 3 years, in :Proceedings of Rehabilitation Engineering Society of Japan, RESJA 2008, pp.241242

Kheir, R.\& Way, T.(2007). Inclusion of deaf students in computer science classes using realtime speech transcription, in: Proceedings of the 12th annual SIGCSE conference on Innovation and technology in computer science education ITiCSE '07, Volume 39 Issue 3, pp.261-265

Lambourne, A.; Hewitt, J.; Lyon, C.\& Warren S.(2004). Speech-Based Real-Time Subtitling Services, International Journal of Speech Technology, Volume 7, Number 4, pp.269 $-279$

McCowan, I.; Moore, D.; Dines J.; Gatica-Perez D.; Flynn M.; Wellner M.\& Boulard H.(2004). On the Use of Information Retrieval Measures for Speech Recognition Evaluation, Institut Dalle Molle d'Intelligence Artificielle Perceptive IDIAP Research Report 0473, pp.1-13

Miyamoto, K.(2005). Effective Master Client Closed Caption Editing System for Wide Range Workforce, in: Proceedings of the Universal Access in Human Computer Interaction Vol. 7, HCI International 2005

Miyamoto, K.(2006). Reduction of Caption Editing Using Supplemental Text Information, Technology \& Persons with Disabilities Conference CSUN 2006

Miyamoto, K.; Arakawa, K.\& Saito, T.(2007). Hybrid Caption Editing System by Integrating Line Unit Type and Word Processor Type. IEICE Transactions on Information and Systems, Vol. J90-D No. 3, pp.673-682

Miyamoto, K.\& Ikawa, Y.(2008). Apparatus and method for rendering contents, containing sound data, moving image data and static image data, harmless, USPTO Application \#: 20080262841, International Business Machines Corporation

Miyamoto, K.\& Shoji, M.(2008). Displaying text of speech in synchronization with the speech, United States Patent 20050203750, International Business Machines Corporation

Miyamoto, K.\& Takizawa, M.(2009). User Efficient Speech Recognition-based Caption Editing System Technology \& Persons with Disabilities Conference CSUN 2009 
Munteanu, C.; Baecker, R.\& Penn, G.(2008). Collaborative Editing for Improved Usefulness and Usability of Transcript-Enhanced Webcasts. in: Proceedings of CHI 2008, ACM Press, pp.373-382

Wald, M.\& Bain, K.(2008). Universal access to communication and learning: the role of automatic speech recognition, in: Universal Access in the Information Society, Vol. 6, No4, pp.435-447 


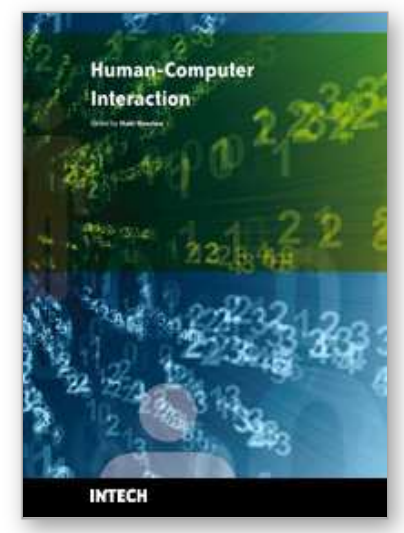

\section{Human-Computer Interaction \\ Edited by Inaki Maurtua}

ISBN 978-953-307-022-3

Hard cover, 560 pages

Publisher InTech

Published online 01, December, 2009

Published in print edition December, 2009

In this book the reader will find a collection of 31 papers presenting different facets of Human Computer Interaction, the result of research projects and experiments as well as new approaches to design user interfaces. The book is organized according to the following main topics in a sequential order: new interaction paradigms, multimodality, usability studies on several interaction mechanisms, human factors, universal design and development methodologies and tools.

\section{How to reference}

In order to correctly reference this scholarly work, feel free to copy and paste the following:

Kohtaroh Miyamoto, Masakazu Takizawa and Takashi Saito (2009). Integration of Speech Recognition-based Caption Editing System with Presentation Software, Human-Computer Interaction, Inaki Maurtua (Ed.), ISBN: 978-953-307-022-3, InTech, Available from: http://www.intechopen.com/books/human-computerinteraction/integration-of-speech-recognition-based-caption-editing-system-with-presentation-software

\section{INTECH}

open science | open minds

\section{InTech Europe}

University Campus STeP Ri Slavka Krautzeka 83/A 51000 Rijeka, Croatia Phone: +385 (51) 770447

Fax: +385 (51) 686166 www.intechopen.com

\section{InTech China}

Unit 405, Office Block, Hotel Equatorial Shanghai No.65, Yan An Road (West), Shanghai, 200040, China 中国上海市延安西路65号上海国际贵都大饭店办公楼405单元 Phone: +86-21-62489820

Fax: +86-21-62489821 
(C) 2009 The Author(s). Licensee IntechOpen. This chapter is distributed under the terms of the Creative Commons Attribution-NonCommercial-ShareAlike-3.0 License, which permits use, distribution and reproduction for non-commercial purposes, provided the original is properly cited and derivative works building on this content are distributed under the same license. 